\title{
Don't Fear Optimality: Sampling for Probabilistic-Logic Sequence Models
}

\author{
Ingo Thon \\ Katholieke Universiteit Leuven \{firstname. lastname\}@cs . kuleuven. be
}

\begin{abstract}
One of the current challenges in artificial intelligence is modeling $d y$ namic environments that change due to the actions or activities undertaken by people or agents. The task of inferring hidden states, e.g. the activities or intentions of people, based on observations is called filtering. Standard probabilistic models such as Dynamic Bayesian Networks are able to solve this task efficiently using approximative methods such as particle filters. However, these models do not support logical or relational representations. The key contribution of this paper is the upgrade of a particle filter algorithm for use with a probabilistic logical representation through the definition of a proposal distribution. The performance of the algorithm depends largely on how well this distribution fits the target distribution. We adopt the idea of logical compilation into Binary Decision Diagrams for sampling. This allows us to use the optimal proposal distribution which is normally prohibitively slow.
\end{abstract}

\section{Introduction}

One of the current challenges in artificial intelligence is modeling dynamic environments that are influenced by actions and activities undertaken by people or agents. Consider modeling the activities of a cognitively impaired person [1]. Such a model can be employed to assist people, using common patterns to generate reminders or detect potentially dangerous situations, and thus can help to improve living conditions. To realize this, the system has to infer the intention or the activities of a person from features derived from sensory information. The typical model used in such processes are Hidden Markov Models (HMM) and their generalizations like factorial HMMs, coupled HMMs or Dynamic Bayesian Networks (DBN). These models can represent the intentions and/or activities with a hidden state. Estimating the hidden state distribution based on a sequence of observation in such model, called filtering, is the task we are focusing on paper.

Algorithms that perform efficient and exact inference in single state models like HMMs are well known. Also, for factorial HMMs and coupled HMMs efficient approximative algorithms exist that exploit structural properties [2] and for DBNs particle filters [3] present a good alternative.

However, recent research has shown that in many activity modeling domains, relational modeling is not only useful [4] [5] but also required [6]. Here, transitions between states are factored into sets of probabilistic logical conjectures that allow a dynamic number of random variables, which makes the translation into a standard Dynamic Bayesian Network impossible. 
Our contributions are: First we show how hidden state inference problems can be formulated through Causal Probabilistic Time Logic (CPT-L). CPT-L was introduced previously, together with the inference and learning algorithms for the fully observable case [7]. We use a logical compilation approach to implement efficient sampling from the optimal proposal distribution. The proposal distribution is a key component of the particle filter algorithm Sequential Importance Resampling (SIR), we want to use in this paper for solving the filtering problem.

Logical compilation has gained lot of interest in the past few years for speeding up inference and learning in probabilistic logics, especially compilation into Binary Decision Diagrams (BDD) [8] [9] [7] annotated with probabilities and its variants Zero Suppressed Decision Diagrams [7] [10] and Algebraic Decision Diagrams [11]. In this work we show as second contribution how a BDD is generated to represent the proposal distribution. Finally we will show as third contribution how the generated BDDs can be used to sample models of the represented formula (the states) according to the underlying distribution.

Related Work: Most sequential SRL models restrict themselves to a single atom per time point [12] or one probabilistic choice, e.g. outcome of an action. We are only aware of the following three exceptions: The Simple Transition Cost Models [13] proposed by Alan Fern. These models allow the specification of costs for transitions, which can be used to represent probabilities, but they have to be equal over all transitions. In Biswas et al.[4] the authors learn a Dynamic Markov Logic Network, but translate to DBNs for inference. Even though this is not a problem in general, it requires a state space with a fixed size that is known in advance. In Zettlemoyer et. al [14], the hidden states are defined by means of weighted FO-Formula the hypothesis. This approach requires mutually exclusive hypotheses, which are hard to construct and it is unclear whether they can deal with functors.

After introducing some terminology we proceed by introducing the CPT-L model. Afterwards, we specify the components of the algorithm that samples from the filtering distribution. Finally we discuss experimental result and conclude.

\subsection{Preliminaries}

Logical representation: A logical atom is an expression $p\left(t_{1}, \ldots, t_{n}\right)$ where $p$ is a predicate symbol of arity $n$. The $t_{i}$ are terms, built from constants, variables and complex terms. Constants are denoted by lower case symbols and variables by upper case symbols. Complex terms are of the form $f\left(t_{1}, \ldots, t_{k}\right)$ where $f$ is a functor symbol and the $t_{i}$ are again terms. An expression is called ground if it does not contain any variables. A substitution $\theta$ maps variables to terms, and $a \theta$ is the atom obtained from $a$ by replacing all variables according to $\theta$. As an example consider the atom $a=p\left(X_{1}, c_{1}, X_{2}\right)$ and the substitution $\theta=\left\{X_{1} \mapsto c_{2}\right\}$ which replaces the variable $X_{1}$ by $c_{2}$ as in $a \theta=p\left(c_{2}, c_{1}, X_{2}\right)$. A set of ground atoms $\left\{a_{1}, \ldots, a_{n}\right\}$ is called Herbrand interpretation used to describe complex states and observations.

Reduced ordered binary decision diagrams: A Boolean formula is a formula build from a set of literals $l_{1}, \ldots, l_{n}$ and the connectors $\wedge$ (and), $\vee$ (or) and $\neg$ (not). Such a function can be represented using a rooted, directed acyclic graph (cf. Figure 1). The terminal nodes correspond to the logical true and false. Each node within the graph 


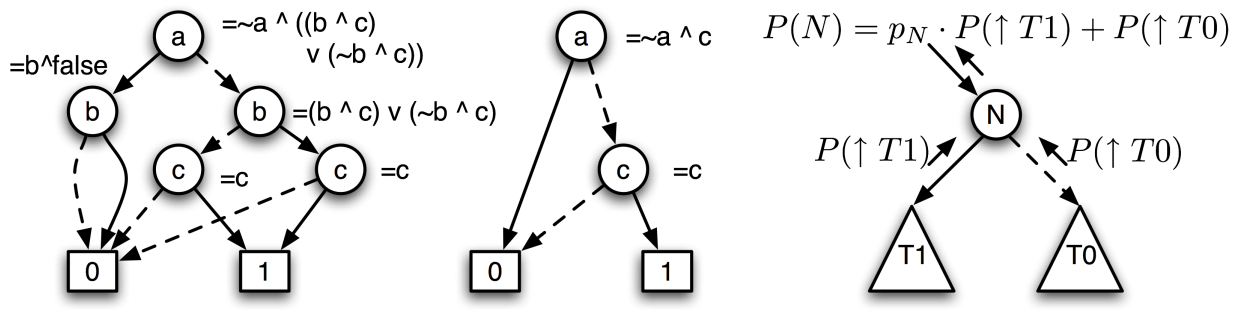

Fig. 1: An ordered Binary Decision Diagram before (left) and after (middle) reducing it. Each node is annotated with the logical formula it represents. For the reduction the $c$ nodes are merged and afterward both $b$ nodes have to be replaced by their child.

Calculation of upward probability (right, cf section 3 ).

corresponds to a literal and has two children one corresponding to assigning the node the value one which is called high-child. The other child corresponds to assigning the value zero called low-child. A reduced ordered binary decision diagram is a decision diagram, where all literals are ordered, isomorphic subtrees are merged and all nodes which have for both branches the same childes are removed. In the following we use the terms BDD and Reduced order BDD synonymously.

\section{Model}

The model considered is basically a HMM where states and observations are Herbrand interpretations and transition- and observation-probabilities are defined in terms of a probabilistic logic (cf. Fig 2). More formally:

Definition 1. A CPT-L model consists of a set of rules of the form

$$
r=\left(h_{1}: p_{1}\right) \vee \ldots \vee\left(h_{n}: p_{n}\right) \leftarrow b_{1}, \ldots, b_{m}
$$

where the $p_{i} \in[0,1]$ form a probability distribution such that $\sum_{i} p_{i}=1, h_{i}$ are logical atoms, $b_{i}$ are literals (i.e. atoms or their negation).

For convenience we will refer to $b_{1}, \ldots, b_{m}$ as body $(r)$ and to $\left(h_{1}: p_{1}\right) \vee \ldots \vee\left(h_{n}: p_{n}\right)$ as $h e a d(r)$. We assume that the rules are range-restricted, that is, all variables appearing in the body also appear in the head.

The interpretation of such a rule is: whenever the body is true at time-point $k$ the rule will cause one of the head elements to be true at time-point $k+1$.

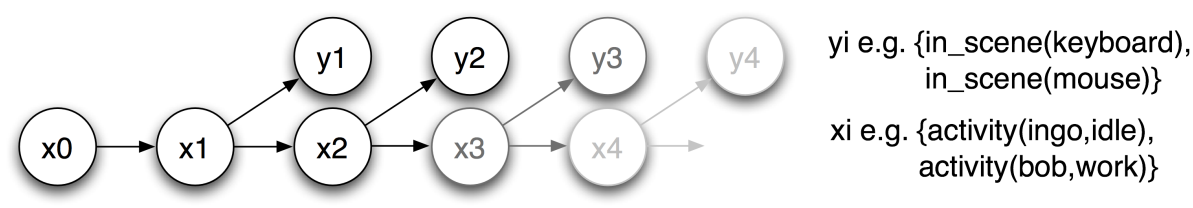

Fig. 2: Graphical representation of an HMM. States and observations are in our case herbrand interpretations. 
Consider the following example rules that models the current activity:

$$
\begin{aligned}
& r(P, X)=a(P, X): 0.8 \vee a(P, \text { drink }): 0.1 \vee a(P, \text { work }): 0.1 \leftarrow a(P, X) . \\
& \operatorname{od}(P)=\quad \text { ois }(\text { can }): 0.7 \vee \text { nil }: 0.3 \quad \leftarrow a(P, \text { drink }) . \\
& \text { ow }(P)=\quad \text { ois }(\text { pen }): 0.7 \vee \text { nil }: 0.3 \quad \leftarrow a(P, \text { work }) \text {. }
\end{aligned}
$$

The first rule states that person $P$ will continue its current activity $X$ with probability 0.8 or switch to one of the activities work, drink with probability 0.1 . The second and third rule specifies: if someone works/drinks one can observe a pen/can.

The semantics of such a model is given by the following probabilistic constructive process. This stochastic process is assumed to be initialized with the empty interpretation or an interpretation containing a special symbol. Starting from the current state, all groundings $r \theta$ of all rules $r$, where body $(r) \theta$ holds in the current state are generated. For each of these grounding, one of the grounded head elements $h_{1} \theta, \ldots, h_{n}$ of $r$ is chosen randomly according to the distribution given by $p_{1}, \ldots, p_{n}$. We will refer to this choice as selection. The chosen head element is then added to the successor state. A more detailed description can be found in [7].

In this work, we additionally assume that a subset of the predicates are declared as observable whereas the others are unobservable. In the previous example the predicate $a / 2$ would typically be unobservable, whereas predicates like pose $/ 2$, movement $/ 2$, object_in_scene/1 (ois/1) would be observable. We assume that all predicates in the head of one rule are either observable or unobservable. A rule is called un-/observable according to the observability of the predicates in the head.

In the rest of the text, $x_{k}$ denotes the set of all unobservable facts and $y_{k}$ the observable facts true at time point $k$. The probability of a hidden state sequence together with a sequence of observations follows directly from the semantics of CPT-L. Note: From the viewpoint of CPT-L the observation $y_{k}$ of time-point $k$ belongs to the successor state as both are caused by the current state. For readability purposes, we keep indexes of hidden states and observations together. With $x_{l: k}$ we denote the sequence of all states between $l$ and $k$.

To continue our example assume that the current state is $x_{i}=\{a($ ann,work $)$, $a(b o b, w o r k)\}$ then the applicable rules are $r(a n n, w o r k), r(b o b$, work $)$, ow (ann), $o d(b o b)$. For each rule a head element is selected and added to the successor state respectively the observation. The state is $x_{i+1}=\{a($ ann, work $), a(b o b, d r i n k)\}$ and the observation is $y_{i}=\{$ ois $($ pen $)\}$ for example with probability $(0.8+0.1) \cdot 0.1$. $(0.7 \cdot(0.3 \cdot 0.7))$.

\section{Algorithm}

Our goal is to estimate the filtering distribution $p\left(x_{k} \mid y_{1: k}\right)$. This distribution can be used to answer queries like $a(P 1, A c t), a(P 2, A c t), P 1 \neq P 2$ whether two persons performing the same activity. Furthermore the distribution can be used for parameter learning with (Stochastic) Expectation Maximization. Exact calculation of this distribution is typically prohibitively slow due to the large structured state space [15]. Therefore we use Sampling Importance Resampling (SIR) [3]: we sample from a proposal distribution and compute importance weights that make up for the difference. In this section 


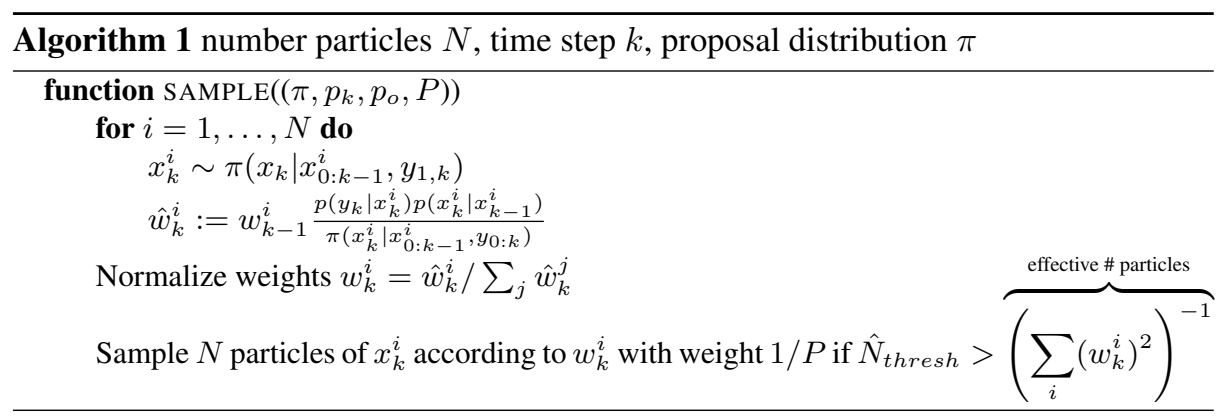

we first briefly discuss the mechanics of SIR. Afterwards we alter the original CPT-L algorithm [7] using a BDD to represent the distributions required by SIR. Finally we give the algorithm to sample states from this distribution using the constructed BDD.

\subsection{Sampling Importance Resampling (SIR)}

The filtering distribution can be approximated by a set of $N$ particles $\left(w_{k}^{i}, x_{k}^{i}\right)$ consisting of a weight $w_{k}^{i}$ and a state $x_{k}^{i}$. The weights are an approximation of the relative posterior distribution. This empirical distribution is defined as

$$
\hat{p}\left(x_{k} \mid y_{1: k}\right)=\frac{1}{N} \sum_{i=1}^{N} w_{i} \delta_{x_{k}^{i}}\left(x_{k}\right),
$$

where $\delta_{x_{k}^{i}}(\cdot)$ is the point mass distribution located at $x_{k}^{i}$.

The SIR algorithm calculates the samples recursively. A single step is described in Algorithm 1. In each step, the particles are drawn from a sampling distribution $\pi\left(x_{k} \mid x_{0: k-1}^{i}, y_{0: k}\right)$ and each particle's weight is calculated. In principle an arbitrary admissible distribution can be chosen as proposal distribution. A distribution is called admissible if it has probability greater zero for each state, which has probability greater zero for the target distribution. So the correctness does not depend on the choice, but the sample variance does, and thus the required number of particles, largely depends on this choice.

Typical sampling distributions are the transition prior $p\left(x_{k} \mid x_{k-1}^{i}\right)$, a fixed importance function $\pi\left(x_{k} \mid x_{0: k-1}^{i}, y_{0: k}\right)=\pi\left(x_{k}\right)$, or the transition posterior $p\left(x_{k} \mid x_{0: k-1}^{i}, y_{0: k}\right)$.

\subsection{Optimal Proposal distribution}

The transition prior $p\left(x_{k} \mid x_{k-1}\right)$ is often used as proposal distribution for SIR as it allows for efficient sampling. Using the transition prior means, on the other hand, that the state space is explored without any knowledge of the observations which makes the algorithm sensitive to outliers. While this nontheless works well in many cases, it is problematic in discrete, high dimensional state spaces when combined with spiked observation distributions. But high dimensional state spaces are common especially 
in relational domains. It can be shown that the proposal distribution $p\left(x_{k}^{i} \mid x_{k-1}^{i}, y_{k}\right)^{\star}$ together with weight update $w_{k}^{i}:=w_{k-1}^{i} P\left(y_{k} \mid x_{k-1}^{i}\right)$ is optimal [3] and does not suffer from this problem.

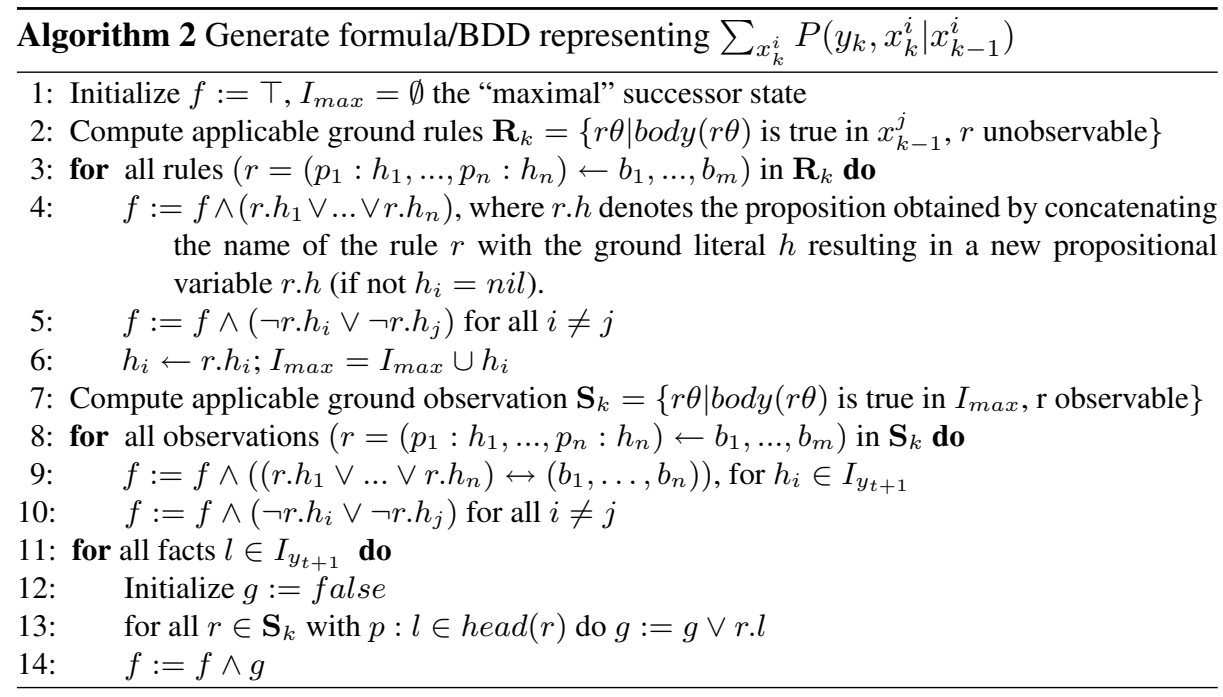

BDD construction: To sample from the proposal distribution and update the weight efficiently we build a BDD that represents $P\left(y_{k} \mid x_{k-1}^{i}\right)$. The algorithm (shown as Algorithm 2) is a modification of the algorithm presented in previous work [7]. The algorithm builds a BDD representation of a formula which computes the joint probability of all possible selections that result in a transition for which the following four conditions hold. The transition (a) starts at $x_{t-1}^{i}$ (line 2) and (b) goes over to a valid successor state $x_{t}$. In $x_{t}$ it (c) generates the observation $y_{t}$ (line 8-14) using (d) the observable rule applicable in $x_{t}$. Each node of the generated BDD $r: h_{i}$ corresponds to selecting (for one rule) the head $h_{i}$ or not, as dictated by the probability $p_{i}$.

BDD sampling Sampling a path according to the $p_{i}$ from the root of this BDD to the terminal node with label 1 corresponds to sampling a value from $p\left(x_{k} \mid x_{k-1}^{i}, y_{k}\right)$. However, in most cases, sampling paths naively according to the $p_{i}$ 's will yield a path ending in the 0-terminal, that will then have to be rejected. Notably this would correspond to sampling from the transition prior. Therefore at every node, when choosing the corresponding subtree, we base our choice not only on its probability, but also on the probability of reaching the 1-terminal through this subtree. This corresponds to conditioning the paths such that we get a valid successor state together with the observation $y_{k}$. We call this probability upward probability because of the way it is computed. The upward probability of the root node corresponds to the probability of reaching the 1terminal, i.e., $P\left(y_{k} \mid x_{k-1}^{i}\right)$. The computation starts by initializing the values in the leafs

* Here it is crucial, to realize that the observation $y_{k}$ is the observation generated by the state $x_{k}$. 


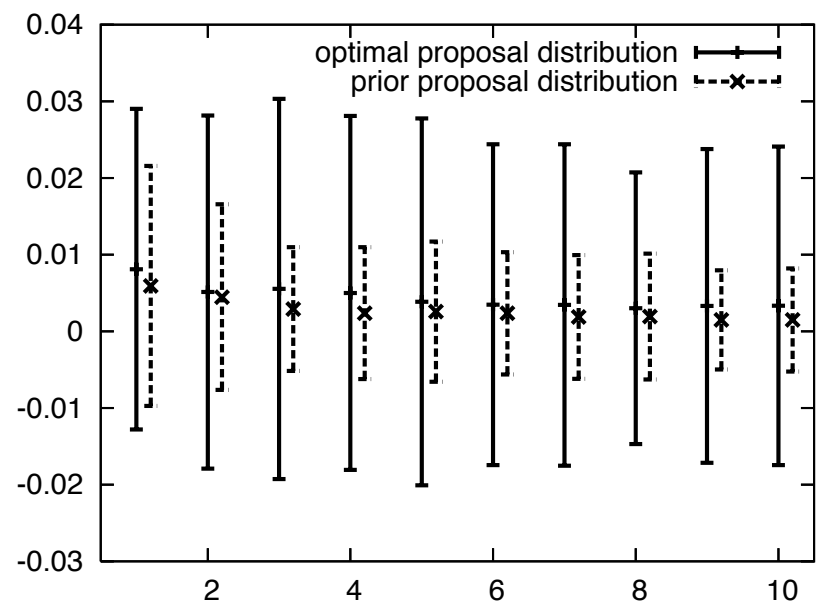

Fig. 3: Effective number of particles divided by runtime in dependence on sequence length.

with the label of the leaf. Afterwards, the probabilities are propagated upward as illustrated in Fig. $1^{\star \star}$. The subtree is then chosen in every node according to

$$
\frac{p_{N} \cdot P\left(\uparrow T 1_{N}\right)}{P\left(\uparrow T 0_{N}\right)+p_{N} \cdot P\left(\uparrow T 1_{N}\right)}=\frac{p_{N} \cdot P\left(\uparrow T 1_{N}\right)}{P(\uparrow N)} \quad \text { respectively: } \frac{P\left(\uparrow T 0_{N}\right)}{P(\uparrow N)} .
$$

\section{Experiments}

To evaluate our algorithm, we recreated the model of Biswas et al [4], according to the parameters specified in their work. There a person is observed during writing, typing, using a mousing, eating, and so on. The computer has multiple cues to classify the activities. Two examples are the pose of the person or whether an apple is observed in the scene. As the observation distribution is fairly smooth and had nowhere zero mass the transition-prior is expected to perform well as proposal distribution.

For the experiments we sampled 5 sequences of length 10. Afterwards we run the particle filter algorithm with the exact proposal distribution and the transition prior using 100 particles. For the optimal prior each run took less then a minute on a MacBook Pro $2.16 \mathrm{Ghz}$. The transition prior was approximately 5 times faster. In Fig 3 the effective number of particles (cf. Alg 1) divided by the runtime in ms is plotted. The horizontal axis is the sequence length. Even though not significant the optimal performed on average better. In toy example with spiked observation distribution the transition prior typically lost all particles in a few steps.

\section{Conclusions and Future work}

We propose a novel way of sampling from a joint distribution in the presence of evidence by the means of BDDs. We show that the final system allows more efficient

\footnotetext{
${ }^{\star \star}$ For the reader familiar with [8] [9] the use here and in [7] is a bit different. The former is the choice of a literal being true or false, whereas the latter represents whether one of the head gets chosen. Using the backward probability of [9] instead of the upward, the sampling generalizes.
} 
filtering than using the transition prior in relational domains. An advantage of our complete system is that the final algorithms are very intuitive as it builds on well established algorithm like SIR. We plan to extend our filtering algorithm towards more elaborate techniques like for example Rao-Blackwellized Particle Filters, and Online Stochastic EM. Finally, we will investigate the use of our technique of sampling from a BDD also for non-sequential probabilistic logics, as well as for standard DBNs.

Acknowledgments: For discussion we are grateful to (in temporal order) K. Kersting, N. Landwehr, L. De Raedt, and B. Gutmann. Special thanks to K. Driessens for approximating a native speaker.

This work was supported by the FWO project: Relational action and activity learning and the GOA/08/008 project "Probabilistic Logic Learning".

\section{References}

1. Pollack, M.E.: Intelligent technology for an aging population: The use of AI to assist elders with cognitive impairment. AI Magazine 26(2) (2005) 9-24

2. Landwehr, N.: Modeling interleaved hidden processes. In: ICML. (2008)

3. Doucet, A., Defreitas, N., Gordon, N.: Sequential Monte Carlo Methods in Practice (Statistics for Engineering and Information Science). 1 edn. Springer (June 2001)

4. Biswas, R., Thrun, S., Fujimura, K.: Recognizing activities with multiple cues. Lecture Notes in Computer Science 4814 (2007) 255

5. Natarajan, S., Bui, H., Tadepalli, P., Kersting, K., Wong, W.K.: Logical hierarchical hidden markov models for modeling user activities. In: ILP. (2008)

6. Sridhar, M., Cohn, A.G., Hogg, D.C.: Learning functional object-categories from a relational spatio-temporal representation. In: ECAI. (2008)

7. Thon, I., Landwehr, N., De Raedt, L.: A simple model for sequences of relational state descriptions. In: ECML. (2008)

8. De Raedt, L., Kimmig, A., Toivonen, H.: Problog: A probabilistic Prolog and its application in link discovery. In: ICJAI. (2007)

9. Ishihata, M., Kameya, Y., Sato, T., Minato, S.: Propositionalizing the EM algorithm by BDDs. In: ILP. (2008)

10. Minato, S.: Compiling bayesian networks by symbolic probability calculation based on zerosuppressed BDDs. In: AAAI. (2007)

11. Chavira, M., Darwiche, A., Jaeger, M.: Compiling relational bayesian networks for exact inference. Int. J. Approx. Reasoning 42(1-2) (2006) 4-20

12. Kersting, K., De Raedt, L., Gutmann, B., Karwath, A., Landwehr, N.: Relational sequence learning. In: Probabilistic Inductive Logic Programming. Volume 4911 of Lecture Notes in Computer Science. Springer (2008) 28-55

13. Fern, A.: A simple-transition model for relational sequences. In: IJCAI. (2005)

14. Zettlemoyer, L.S., Pasula, H.M., Kaelbling, L.P.: Logical particle filtering. In: Probabilistic, Logical and Relational Learning - A Further Synthesis. (2008)

15. Boyen, X., Koller, D.: Tractable inference for complex stochastic processes. In: UAI. (1998) 